\title{
Cell Permeability of Isomeric Macrocycles: Predictions and NMR Studies
}

Fabio Begnini,, Vasanthanathan Poongavanam,, Yoseph Atilaw, Mate Erdelyi, Stefan Schiesser, and Jan Kihlberg*

Cite This: ACS Med. Chem. Lett. 2021, 12, 983-990

Read Online

ACCESS I

Llll Metrics \& More

Article Recommendations

Supporting Information

ABSTRACT: Conformation-dependent 3D descriptors have been shown to provide better predictions of the physicochemical properties of macrocycles than $2 \mathrm{D}$ descriptors. However, the computational identification of relevant conformations for macrocycles is nontrivial. Herein, we report that the Caco2 cell permeability difference between a pair of diastereomeric macrocycles correlated with their solvent accessible 3D polar surface area and radius of gyration. The descriptors were calculated from the macrocycles' solutionphase conformational ensembles and independently from ensembles obtained by conformational sampling. Calculation of the two descriptors for three other stereo- and regioisomeric macrocycles also allowed the correct ranking of their cell permeability. Methods for conformational sampling may thus allow ranking of passive permeability for moderately flexible macrocycles, thereby contributing to the prioritization of macrocycles for synthesis in lead optimization.

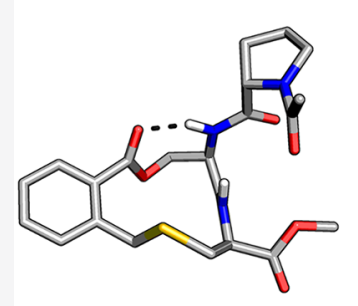

1 (Conf. 7, 29\%)

$3 \mathrm{D} P S A=114 \AA^{2}$

$\mathrm{R}_{\mathrm{gyr}}=3.98 \AA$

More permeable $Y$

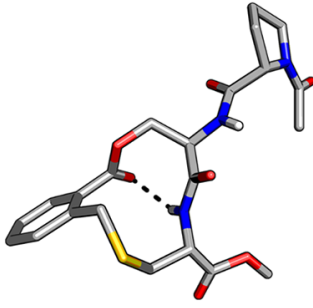

2 (Conf. 4, 28\%)

3D PSA $=154 \AA^{2}$

$\mathrm{R}_{\mathrm{gyr}}=4.42 \AA$

Less permeable $X$

KEYWORDS: Cell permeability, conformational sampling, macrocycle, NMR spectroscopy

M acrocycles are of major interest in efforts to discover drugs for targets which are challenging to modulate with rule of 5 (Ro5) compliant compounds. ${ }^{1-3}$ In particular, the ability of macrocycles to adopt disk- and sphere-like shapes make them ideal ligands for targets that have large, flat, or groove-shaped binding sites. ${ }^{2}$ It is essential that macrocycles are cell permeable in order to reach intracellular difficult-todrug targets, such as protein-protein interactions. Cell permeability is also required for compounds to be absorbed after oral administration. ${ }^{4}$ Interestingly, some studies have found that macrocyclization can result in major increases in cell permeability, ${ }^{5,6}$ while others have found only a limited increase $^{7,8}$ or a reduction in permeability upon macrocyclization. ${ }^{9}$

Macrocyclic drugs often require long synthetic routes, and the macrocyclization step may be accompanied by low yields or require significant optimization. ${ }^{10}$ Methods for the prediction of pharmacokinetic properties, such as cell permeability, are therefore of significant interest to reduce the synthesis of macrocycles outside the desired property space. Recent studies have concluded that $2 \mathrm{D}$ descriptors for lipophilicity and polarity, for example, cLogP and the topological polar surface area (TPSA), that are often used for design of Ro5 compliant compounds are less useful for macrocycles in particular when their size, structural complexity, and flexibility increases. ${ }^{11-14}$ Instead, knowledge of the 3D structures, conformational preferences, and intramolecular interactions such as hydrogen bonds has been suggested to be essential for better prediction of cell permeability. ${ }^{13-15}$ However, prediction of biologically relevant conformations of complex macrocycles is far from trivial. ${ }^{14,16}$ Some promising progress has been made for small sets of macrocyclic peptides, ${ }^{5,17,18}$ and natural-product-inspired macrocycles, ${ }^{14,19,20}$ albeit by using substantial time and computational resources for each compound. However, in order to impact on the prioritization of compounds for synthesis in lead optimization, medium- to high-throughput methods that are amenable to automation are desired.

Diastereomers share the same 2D descriptors but may differ in cell permeability and other properties that influence oral absorption. ${ }^{19,21}$ Predicting property differences displayed by diastereomers therefore requires use of descriptors derived from their conformations. ${ }^{14}$ Consequently, diastereomers provide ideal opportunities to evaluate methods for generation of structure-property relationships for compounds having complex structures. We recently discovered four diastereomeric macrocycles that display a significant difference in

Received: March 2, 2021

Accepted: May 13, 2021

Published: May 18, 2021 
permeability across human colon adenocarcinoma (Caco-2) cell monolayers in a project aimed at finding inhibitors of the Keap1-Nrf2 protein-protein interaction. ${ }^{22}$ Herein, we report the determination of the solution conformational ensembles of the two macrocycles that differed most in permeability. We also report that descriptors calculated from the independent prediction of the conformational ensembles of these two macrocycles ranked their permeability correctly.

The Caco- 2 cell line has been used as an in vitro model of oral absorption in humans for over 30 years. ${ }^{23}$ Diastereomeric macrocycles $1-4,{ }^{22}$ which all contain $S$-Pro but differ in the stereochemistry of the Ser and Cys moieties, displayed differences in their passive, efflux inhibited permeabilities. Macrocycle 1 had the highest permeability, which was roughly 7 -fold higher than that of 2, whereas macrocycles 3 and 4 had intermediate permeabilities. As macrocycles 1-4 display no or only minor differences in their measured $\log \mathrm{D}_{7.4}$ values, NMR studies and conformational sampling were performed for $\mathbf{1}$ and 2 to investigate the reason for their permeability difference (Figure 1).

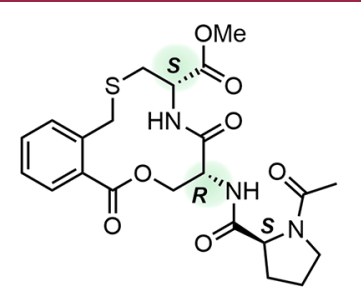

1

Perm $=5.6 \pm 1.2$

$\log _{7.4}=0.65 \pm 0.05$

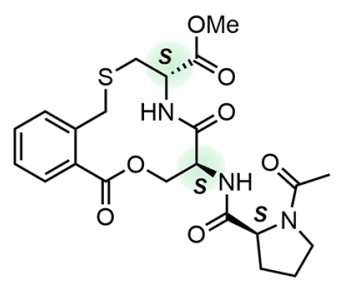

3

Perm $=2.9 \pm 1.9$

$\log _{7.4}=0.60 \pm 0.08$

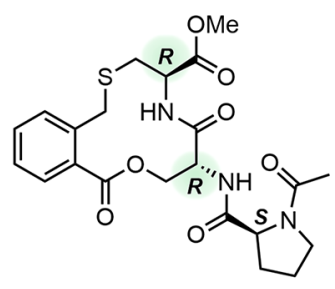

2

Perm $=0.8 \pm 0.3$

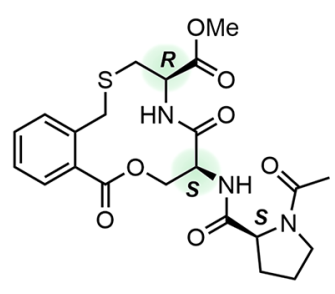

4

Perm $=3.5 \pm 1.0$

$\log _{7.4}=0.43 \pm 0.05$ $\log _{7.4}=0.70 \pm 0.00$

Figure 1. Structures of macrocycles $1-4$ as well as their passive permeability across a Caco-2 cell monolayer (Perm: $\mathrm{P}_{\text {app }} \mathrm{AB}+$ inhibitor cocktail $\left.\times 10^{-6} \mathrm{~cm} / \mathrm{s}\right)$ and their lipophilicity $\left(\log \mathrm{D}_{7.4}\right)$ determined at $\mathrm{pH}$ 7.4. Standard errors were obtained from four and five repeats for $\mathbf{1}$ $\left(\log \mathrm{D}_{7.4}\right.$ and Caco-2, respectively) and three repeats for each assay for $2-4$.

The conformations adopted in a low dielectric medium can be used to predict permeability differences between compounds. ${ }^{13-15,18,19}$ Consequently, we determined the conformational ensembles of macrocycles $\mathbf{1}$ and $\mathbf{2}$ in chloroform, which has a similar dielectric constant $(\varepsilon=4.8)$ to that of the interior of a lipid bilayer $(\varepsilon=3.0) .{ }^{24}$ As 1 and 2 are expected to exist as ensembles of rapidly interconverting conformations, we used the NMR analysis of molecular flexibility in solution (NAMFIS) algorithm ${ }^{25}$ to deconvolute population averaged NMR data into individual conformations. Previously, this algorithm has been applied to determine the solution ensembles of small molecules, peptides, and macrocycles. $^{15,16,26,27}$
By the use of nuclear Overhauser effects (NOEs), scalar coupling constants, and theoretical conformational ensembles as inputs, NAMFIS generates the ensemble that best fits to the experimental observations (Tables S2-S5, Figure S2). NOEs were quantified from a NOESY buildup with at least four spectra using the initial rate approximation. A pool of theoretically feasible conformers were generated by Monte Carlo conformational searches (Table S6) using five different force fields combined with implicit chloroform and water solvation models for each search, to ensure that the conformational space available for the compounds is sampled efficiently. ${ }^{15}$ For both $\mathbf{1}$ and 2 , the 10 output ensembles were merged, and redundant conformers were eliminated using a 1 Å root-mean-square deviation (RMSD) cutoff (Table S6). The conformations adopted by the macrocyclic core of $\mathbf{1}$ and $\mathbf{2}$ are well-described both by the NMR data and the theoretical conformational search, whereas the orientations of the proline and methyl ester side-chains could not be defined in the NAMFIS analysis because of an insufficient number of experimental data (Tables S2-S5, Figure S2). For the proline moiety of 1 and 2, the orientations of the Ser $\mathrm{C}_{\alpha}-\mathrm{NH}$ bond were defined by the $\mathrm{H}_{\alpha}-\mathrm{NH}$ coupling constant and by two NOEs between the Ser $\mathrm{NH}$ and the hydrogen atoms of the macrocyclic core. However, no or only one experimental restraint was found for the Pro $\mathrm{C}_{\alpha}-\mathrm{CONH}$ and amide bond, just as for the bonds of Cys methyl ester. The lowest energy (OPLS2) conformation about these bonds was therefore predicted by torsional scans conducted with 12 rotamers and $30^{\circ}$ angle increments per bond.

The conformational ensemble of 1 consisted of seven conformations (Figure 2A, Table S9). Pairwise RMSD values for all heavy atoms (i.e., all non-hydrogen atoms) in the seven conformations ranged from 0.97 to $2.96 \AA$ (Table S12), while the pairwise RMSD values of heavy atoms of the macrocyclic core ranged from 0.36 to $0.90 \AA$ (Table S10). One conformational family (conformations 1, 3 and 7) and four distinct conformations were identified using a heavy atom RMSD cutoff of $0.5 \AA$ for the heavy atoms in the macrocyclic core. Conformations 1,3 and 7 also have the proline and methyl ester side chains in similar orientations where the proline moiety is oriented toward the macrocyclic ring. However, the orientation of the carbonyl oxygen of the lactone differ between the three conformations in the family. The macrocycle as well as the side chains in conformation 2 display similarities with conformation 1 but differ from conformations 3 and 7 in the family. In contrast, conformations 4-6 differ significantly from the other four and between each other, both for the macrocyclic core and for the orientation of the two side chains. Conformation 4 does not show any intramolecular hydrogen bond (IMHB), whereas the other six conformations possess either one or two IMHBs (Figure 2A, Table S14).

Macrocycle 2 also populates seven conformations that show a similar structural diversity as the members of the ensemble of macrocycle 1 (Figure 2B, Table S9). Pairwise RMSD values for all heavy atoms of 2 range from 1.55 to $3.02 \AA$ (Table S13), while the RMSD values for macrocycle core range from 0.33 to $0.99 \AA$ (Table S11). Conformations 2 and 3 cluster into one family when using a heavy atom RMSD cutoff of $\leq 0.5 \AA$ for the macrocyclic core. These two conformations share the shape of the macrocycle but differ somewhat in the orientation of the amide bond within the macrocyclic core and in the orientation of the two side-chains. The macrocycle ring in 
A

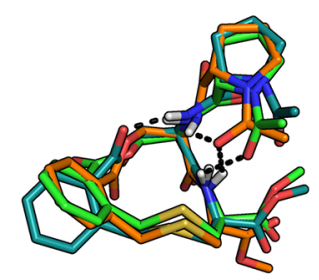

Conf. 1 (green) - 10\%

Conf. 3 (orange) - 2\%

Conf. 7 (dark green) - 29\%

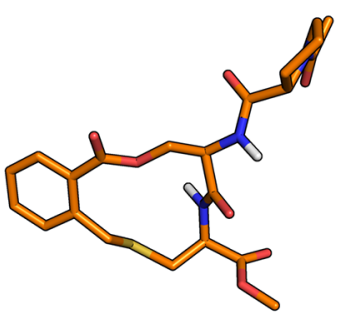

Conf. 4 - 9\%

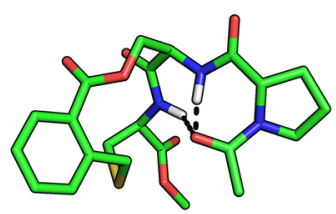

Conf. $6-17 \%$
B

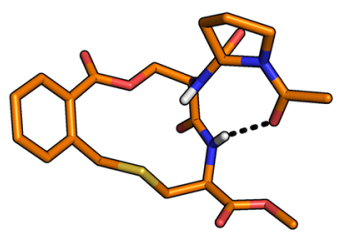

Conf. 2 - 2\%

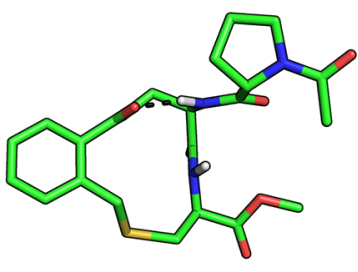

Conf. 5 - 29\%

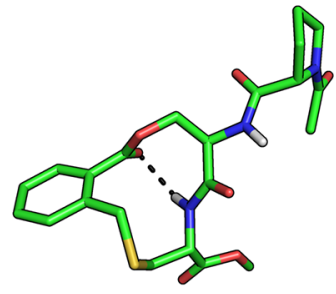

Conf. 4 - 28\%

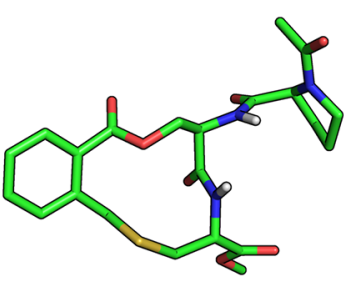

Conf. $6-18 \%$

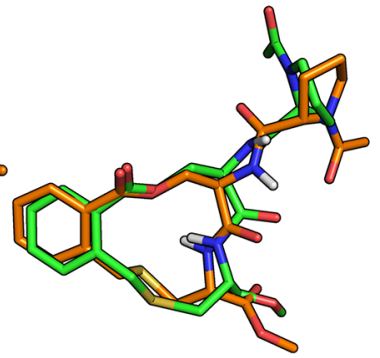

Conf. 2 (green) - 11\% Conf. 3 (orange) - 6\%

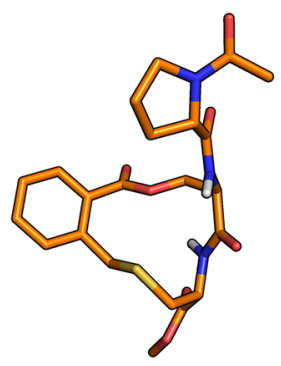

Conf. 5 - 3\%

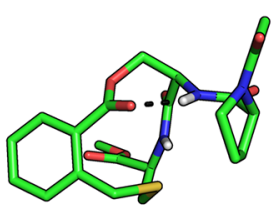

Conf. $7-26 \%$

Figure 2. Conformational ensembles of macrocycles 1 (A) and 2 (B) in $\mathrm{CDCl}_{3}$ determined by NAMFIS analysis. Major conformations (relative population $\geq 10 \%$ ) are colored in green; minor conformations (relative population $<10 \%$ ) are in orange. Conformations that have an RMSD $\leq 0.5$ $\AA$ for the heavy atoms in the macrocyclic core have been superimposed on the basis of these atoms. IMHBs are indicated as black dashed lines. Nonpolar hydrogen atoms have been omitted for clarity.

conformation 1 has similarities to the ring in conformations 2 and 3 , whereas the macrocycles in the remaining conformations (4-7) are distinct from each other and from 1-3 (Figure 2B, Table S11). Single IMHBs are formed in three (1, 4, and 7) of the conformations of 2 (Figure 2B, Table S14).

We performed conformational sampling of $\mathbf{1}$ and $\mathbf{2}$ to investigate how well the sampled ensembles resembled the experimental ones obtained by NAMFIS analysis and if the sampled ensembles could be used to predict the permeability difference between $\mathbf{1}$ and $\mathbf{2}$. Conformational sampling was performed using OMEGA ${ }^{28}$ starting from the SMILES (simplified molecular-input line-entry system) codes of $\mathbf{1}$ and 2 in implicit chloroform $(\varepsilon=4.8)$. An energy window of 10 $\mathrm{kcal} / \mathrm{mol}$ was used to evaluate to what extent OMEGA identified conformations similar to the experimentally determined ones. Conformations obtained from sampling were energy minimized using the molecular mechanics force field MMFF94. ${ }^{29}$ The number of conformations identified by sampling differed significantly between macrocycles $\mathbf{1}$ and 2; eight conformations were found for $\mathbf{1}$ and 28 for 2 .

Sampling of biologically relevant conformations of macrocycles is far from trivial. ${ }^{16,30}$ Therefore, comparisons of the experimentally determined and sampled ensembles of $\mathbf{1}$ and $\mathbf{2}$ were made for all heavy atoms (Figure 3A,B) and for all heavy atoms in the macrocyclic core (Figure 3C,D). Using an RMSD of $\leq 1.0 \AA$ as the cutoff for high similarity for the whole macrocycle revealed that none of the sampled conformations of $\mathbf{1}$ and $\mathbf{2}$ reproduced the experimental conformations with high similarity (Figure 3A,B). However, for 1 the sampled conformations reproduced five of the seven experimental conformations (numbers 1, 3, 5-7; 89\% of the ensemble) with intermediate similarity $(1.0-1.5 \AA)$, with the sampled minimum energy conformation (MEC) being similar to three of the experimental conformations. For 2, the sampled conformations reproduced four of the seven experimental conformations (numbers 1, 3, 4 and $6 ; 58 \%$ of the ensemble) with intermediate similarity, but at energies $>5 \mathrm{kcal} / \mathrm{mol}$ above the MEC. A principle moment of inertia (PMI) plot revealed that experimental and sampled conformations of $\mathbf{1}$ and $\mathbf{2}$ adopted similar rod to sphere-like shapes (Figure S4). Using an RMSD $\leq 0.5 \AA$ as cutoff for high similarity revealed that the structure of the macrocyclic core in all but conformation 4 (9\%) of 1 , and conformations $1(6 \%)$ and $2(11 \%)$ of 2 , were reproduced by at least one of the sampled conformations (Figure 3C,D). Interestingly, three of the core conformations in each of the ensembles of $\mathbf{1}$ and 2, which represent 75 and $37 \%$ of each ensemble, where reproduced with excellent similarity (RMSD $\leq 0.1 \AA$ A). However, most experimental 


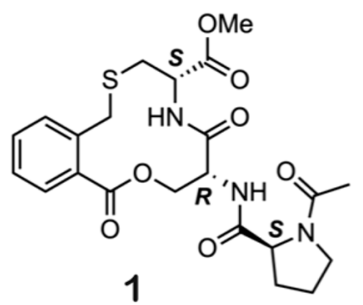<smiles>[R]C(CSCc1ccccc1C(=O)OC)NC(=O)C(=O)NC(=O)C1CCCN1C(C)=O</smiles>
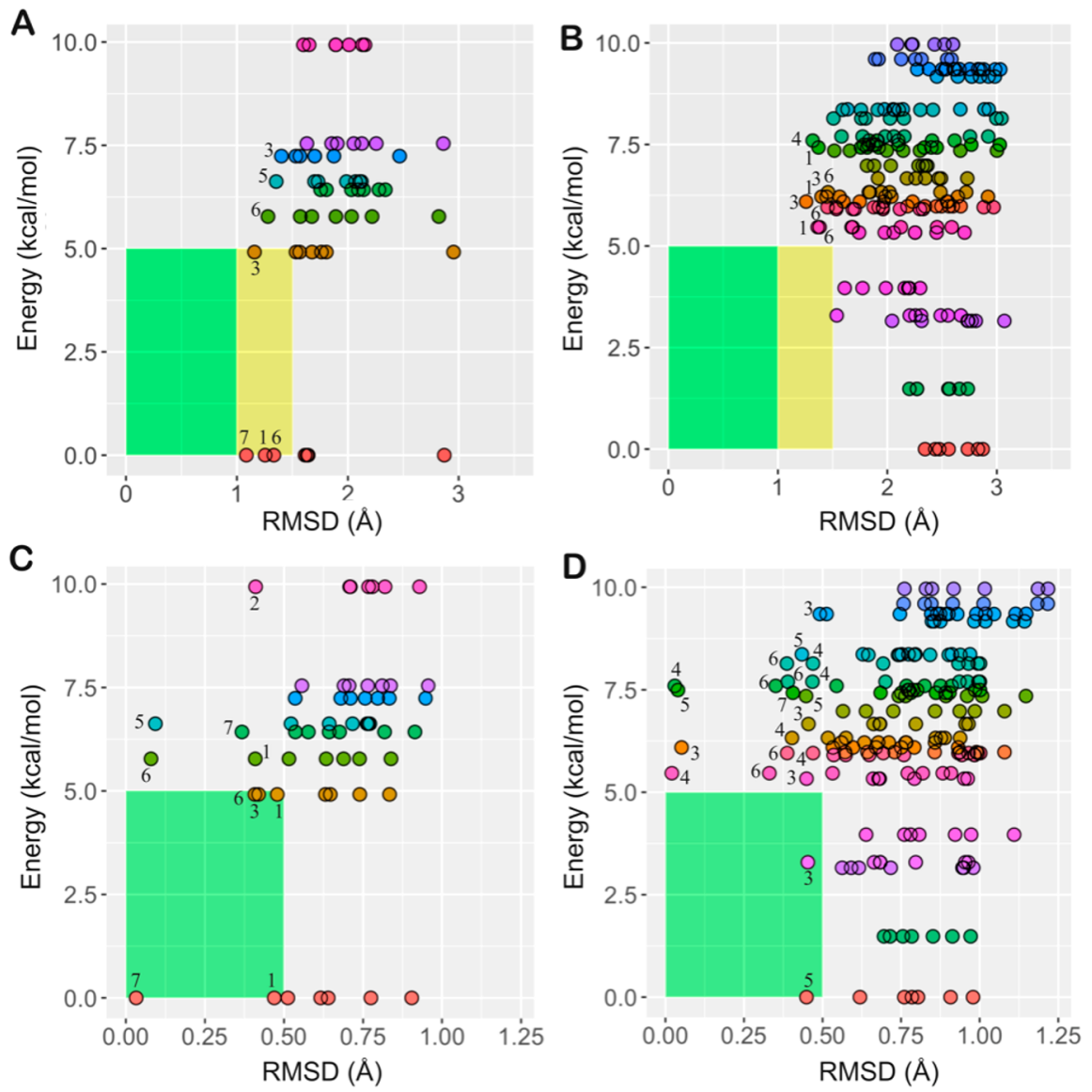

Figure 3. Comparison of all heavy atoms (A and B), or the heavy atoms of the macrocyclic core (C and D), in the sampled conformations of $\mathbf{1}$ and 2 to the experimentally determined conformations. In each panel, the sampled conformations are arranged by increasing energy above the minimum energy conformation (MEC), as indicated by the horizontal rows of colored circles. The circles indicate how similar (by RMSD) the sampled conformation at that energy is to each of the seven experimentally determined conformations of $\mathbf{1}$ or $\mathbf{2}$. In panels A and B, the numbers of all experimental conformations that are similar (RMSD $\leq 1.5 \AA$, for all heavy atoms in $\mathbf{1}$ and $\mathbf{2}$ ) to the sampled conformations are given adjacent to the corresponding colored circle. In panels $\mathrm{C}$ and $\mathrm{D}$, the numbers of all experimental conformations in which the macrocyclic core is similar (RMSD $\leq 0.5 \AA$, for all heavy atoms in the macrocyclic ring of 1 and 2) to the ring in the sampled conformations are given adjacent to the corresponding colored circle. In panels A-D, the space in which the experimental and sampled conformations have a high similarity (RMSD $\leq 1.0$ Å for all heavy atoms, RMSD $<0.5 \AA$ for the heavy atoms in the macrocyclic core) within $5 \mathrm{kcal} / \mathrm{mol}$ of the MEC is highlighted in green. Similarly, in panels $\mathrm{A}$ and $\mathrm{B}$, the space in which the experimental and sampled conformations have medium similarity (RMSD 1.0-1.5 $\AA$ for all heavy atoms) within $5 \mathrm{kcal} / \mathrm{mol}$ of the MEC is highlighted in yellow.

conformations were reproduced by sampled conformations having energies close to or $>5 \mathrm{kcal} / \mathrm{mol}$ above the MECs. Only the core of conformation 7 of macrocycle 1 was reproduced with excellent similarity by the sampled MEC. Thus, sampling was less successful in generating ensembles in which the sidechains adopted correct conformations than in identifying the solution conformations of the macrocyclic cores. In addition, sampled conformations that were similar to the experimentally determined ones were usually found at energies $\geq 5 \mathrm{kcal} / \mathrm{mol}$ above the MEC. This, again, illustrates the difficulty of force fields, such as MMFF94, to rank the conformations for macrocycles by energy so that the major solution conformations are identified. ${ }^{14,16}$
Recently, we suggested that physicochemical properties of macrocycles are better assessed by molecular descriptors, such as the radius of gyration $\left(R_{\text {gyr }}\right)^{31}$ and solvent accessible $3 \mathrm{D}$ polar surface area (SA 3D PSA) ${ }^{13,32}$ than by energy- or RMSDbased criteria. ${ }^{16}$ We therefore calculated the $R_{\text {gyr }}$ and SA 3D PSA for both the experimentally determined and sampled ensembles of $\mathbf{1}$ and $\mathbf{2}$ to investigate if these descriptors correlate with the observed permeability difference between the compounds. $R_{\mathrm{gyr}}$ is suitable to describe differences in size between diastereomers as it depends on the $3 \mathrm{D}$ structure and conformation(s) of a compound. The topological polar surface area (TPSA) is a satisfactory descriptor of polarity for Ro5 compliant compounds ${ }^{33}$ but does not account for differences 
between diastereomers. For these, it is better to use the solvent-accessible 3D PSA ${ }^{32}$ (SA 3D PSA) and to include partially charged atoms in addition to nitrogen, oxygen, and attached hydrogen atoms ${ }^{34,35}$ in the calculations. ${ }^{13}$

The $R_{\text {gyr }}$ was calculated for the experimentally determined conformations of macrocycles $\mathbf{1}$ and $\mathbf{2}$, as well as for the sampled ensembles (Figure 4, Tables S14-S15). The

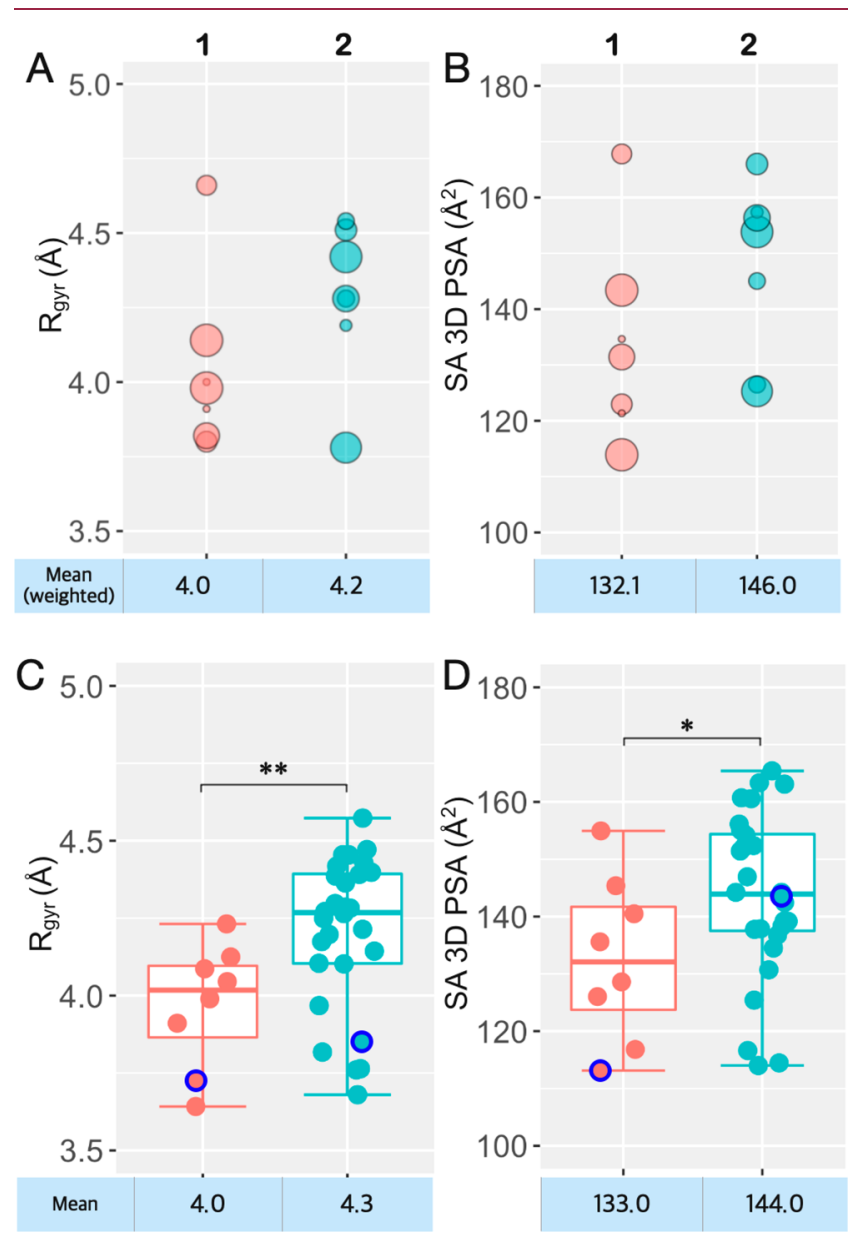

Figure 4. (A) Calculated radius of gyration $\left(R_{\text {gyr }}\right)$ and (B) solventaccessible 3D polar surface area (SA 3D PSA) for the experimentally determined conformations of macrocycles 1 and 2 . The size of each circle is proportional to the relative population of each conformer. (C) Calculated radius of gyration $\left(R_{\text {gyr }}\right)$ and $(D)$ solvent-accessible 3D polar surface area (SA 3D PSA) for the sampled conformational ensembles of macrocycles $\mathbf{1}$ and $\mathbf{2}$. Boxplots show the 50th percentiles as horizontal bars, the 25th and 75th percentiles as boxes, the 25th percentile minus $1.5 \times$ the interquartile range and the 75 th percentile plus $1.5 \times$ the interquartile range as whiskers. The minimum energy conformations (MECs) are indicated as blue circles. Population weighted mean values are given below panels $\mathrm{A}$ and $\mathrm{B}$, while mean values are given below panels $\mathrm{C}$ and $\mathrm{D}$. Wilcoxon test $p$-values: $* \leq$ $0.05, * * \leq 0.01$.

population weighted mean $R_{\text {gyr }}$ is somewhat smaller for the experimental conformations of macrocycle 1 than for 2 (4.04 vs $4.23 \AA$ ). Even though this difference may not have a major impact on the observed difference in permeability, it is interesting to note that the mean $R_{\mathrm{gyr}}$ for the sampled conformational ensembles of 1 and 2 (4.01 vs $4.27 \AA$ ) agreed well with the values obtained for the experimentally determined ensembles. Calculation of the SA 3D PSA revealed that the population weighted mean SA 3D PSA was significantly lower for the experimentally determined ensemble of 1 than for the ensemble of 2 (132 vs $146 \AA$, Figure 4B). This agrees well with the ensemble of 1 showing a higher degree of intramolecular hydrogen bonding than that of 2 (Figure 2, Table S14). The SA 3D PSA for the sampled conformational ensembles of 1 and 2 (means; 134 vs $144 \AA$ ) showed an excellent agreement with the values from the experimental ensembles, just as was found for $\mathrm{R}_{\mathrm{gyr}}$. In conclusion, the experimental ensemble of $\mathbf{1}$ is shifted toward lower values for both $R_{\text {gyr }}$ and SA 3D PSA than the ensemble of 2 , in full agreement with 1 displaying a higher passive Caco-2 cell permeability than 2 . Importantly, these differences between 1 and 2 were also well predicted by the ensembles sampled by OMEGA, suggesting that calculated descriptors from conformational sampling could be used for prospective ranking of cell permeability.

In addition, we investigated if predicted 3D descriptors may be used for prospective ranking of cell permeability using macrocycles $5-7,{ }^{19}$ which include a regioisomeric ( 5 and 7$)$ and a diastereomeric (6 and 7) matched pair that differ in passive cell permeability (Figure 5A) but have identical calculated 2D descriptors (Table S17). Sampled ensembles were generated for 5-7, and then $R_{\mathrm{gyr}}$ and SA 3D PSA were calculated for the conformers, using the same protocols as for $\mathbf{1}$ and 2. Encouragingly, the calculated values of the two 3D descriptors agreed well with the permeabilities of 5-7, that is, the least permeable regioisomer $\mathbf{5}$ had a significantly higher

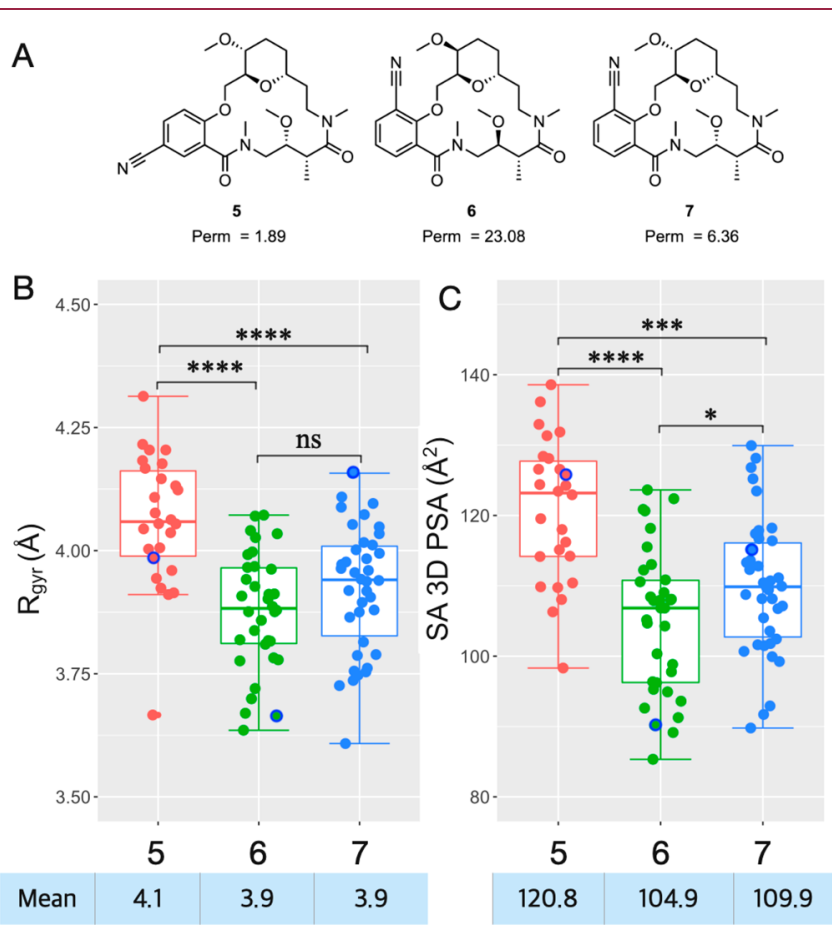

Figure 5. (A) Structures of macrocycles 5-7 and their passive permeability across a Caco-2 cell monolayer ${ }^{19}$ (Perm: $P_{\text {app }} A B$ +inhibitor cocktail $\times 10^{-6} \mathrm{~cm} / \mathrm{s}$ ). (B) Calculated radius of gyration $\left(R_{\mathrm{gyr}}\right)$ and $(\mathrm{C})$ solvent-accessible 3D polar surface area (SA 3D PSA) for the sampled conformational ensembles of 5-7. Boxplots show the 50 th percentiles as horizontal bars, the 25 th and 75 th percentiles as boxes, the 25 th percentile minus $1.5 \times$ the interquartile range and the 75 th percentile plus $1.5 \times$ the interquartile range as whiskers. The minimum energy conformations (MECs) are indicated as blue circles, and mean values are given below the panels. Wilcoxon test $p$-values: * $\leq 0.05, * * \leq 0.01, * * * \leq 0.001, * * * * \leq 0.0001$. 
$R_{\text {gyr }}$ and SA 3D PSA than stereoisomers 6 and 7 (Figure 5B,C). There was also a difference in SA 3D PSA between 6 and 7 that matched the lower permeability of 7 . The less permeable regioisomer 5 adopted more rod-like conformations than the sphere-like 6 and 7 (Figure S4).

The flexibility of a compound is another key determinant of its oral bioavailability and cell permeability. ${ }^{36,37}$ Kier's flexibility index $(\Phi)^{38}$ has been highlighted to provide a better description of the flexibility of macrocyclic compounds ${ }^{36}$ than the number of rotatable bonds (NRotB), ${ }^{37}$ which is calculated from single bonds that are not part of a ring. Potentially, use of $\Phi$ to characterize the flexibility of the macrocyclic ring, in combination with NRotB for the flexibility of the side chains, could be the preferred approach for macrocyclic compounds. Calculations using the MOE software revealed that $\mathbf{1}$ and 2 had NRotB $=6$ and $\Phi=7.12$, while 5-7 had NRotB $=3$ and $\Phi=9.32$. A value of 10 for $\Phi$ was recently proposed as an upper limit within which it could be possible to predict macrocycle cell permeability based on conformational sampling. ${ }^{14}$ It is therefore interesting to note that descriptors calculated for conformational ensembles of 1 and 2, and 5-7, successfully ranked their permeabilities.

The 3D structure and conformational preferences of compounds that have high structural complexity and/or flexibility are key determinants of their cell permeability. ${ }^{13,19,36}$ Prediction of properties from relevant conformational ensembles would therefore be useful in lead optimization of such compounds. Herein, we found that descriptors, that is, the solvent accessible 3D polar surface area (SA 3D PSA) and radius of gyration $\left(R_{\text {gyr }}\right)$, calculated from the conformational ensembles determined by NMR spectroscopy in chloroform correlated with the difference in Caco- 2 cell permeability between diastereomeric macrocycles $\mathbf{1}$ and 2 . This reiterates the relevance of conformation-dependent descriptors for prediction of macrocycle cell permeability. ${ }^{13,32}$ Conformational sampling reproduced the conformations of the macrocyclic cores in the experimental ensembles of $\mathbf{1}$ and $\mathbf{2}$ with high or excellent accuracy (RMSD $0.1-0.5 \AA$ ), but the conformations of the overall compounds were only reproduced with intermediate accuracy (RMSD 1.0-1.5 $)$ ). However, as previously observed, ${ }^{16}$ the energies of the relevant sampled conformations were usually high above the minimum energy conformation, preventing an energy-based identification of the permeating conformations. Interestingly, the mean values of the SA 3D PSA and $R_{\text {gyr }}$ for the sampled ensembles of 1 and 2 were in excellent agreement with the corresponding values for the experimental ensembles. This indicates that $3 \mathrm{D}$ descriptors from sampled ensembles may be of use for prospective ranking of cell permeability; a hypothesis that was supported by the predicted SA 3D PSA and $R_{\text {gyr }}$ of the stereo- and regioisomeric macrocycles 5-7. In a recent analysis, we postulated that conformational sampling was likely to provide successful rankings of the permeability for compounds possessing a Kier flexibility index $(\Phi)<10 .^{14}$ Our current findings agree well with these results as macrocycles 1 and 2 have $\Phi=7.12$ and 5-7 have $\Phi=9.32$. Medium-throughput methods for conformational sampling may thus allow ranking of passive permeability for moderately flexible macrocycles. Incorporation of such methods in compound design during the lead optimization phase should help medicinal chemists to select and synthesize only the macrocycles which are within the desired property space.

\section{ASSOCIATED CONTENT}

\section{Supporting Information}

The Supporting Information is available free of charge at https://pubs.acs.org/doi/10.1021/acsmedchemlett.1c00126.

NMR data; Monte Carlo molecular mechanics conformational search and NAMFIS conformational analysis of macrocycles 1 and 2; computational characterization of 1, 2, and 5-7; procedures for determination of $\log \mathrm{D}_{7.4}$ and Caco-2 cell permeability; purity analyses for 1-4; NMR spectra of 1 and 2 (PDF)

\section{AUTHOR INFORMATION}

\section{Corresponding Author}

Jan Kihlberg - Department of Chemistry - BMC, Uppsala University, 75123 Uppsala, Sweden; (1) orcid.org/00000002-4205-6040; Email: jan.kihlberg@kemi.uu.se

\section{Authors}

Fabio Begnini - Department of Chemistry - BMC, Uppsala University, 75123 Uppsala, Sweden

Vasanthanathan Poongavanam - Department of Chemistry BMC, Uppsala University, 75123 Uppsala, Sweden; (1) orcid.org/0000-0002-8880-9247

Yoseph Atilaw - Department of Chemistry - BMC, Uppsala University, 75123 Uppsala, Sweden

Mate Erdelyi - Department of Chemistry - BMC, Uppsala University, 75123 Uppsala, Sweden; orcid.org/00000003-0359-5970

Stefan Schiesser - Department of Medicinal Chemistry, Research and Early Development, Respiratory and Immunology (R\&I), BioPharmaceuticals R\&D, AstraZeneca, 43183 Mölndal, Sweden; 다잉.org/0000-0002-86682844

Complete contact information is available at:

https://pubs.acs.org/10.1021/acsmedchemlett.1c00126

\section{Author Contributions}

${ }^{\#}$ F.B. and V.P. made equal contributions to the manuscript. This manuscript was written through contributions of all authors. All authors have approved the final version of the manuscript.

\section{Funding}

This work was funded by grants from AstraZeneca and the Swedish Research Council (grant no. 2016-05160; J.K. and 2016-03602; M.E.).

\section{Notes}

The authors declare the following competing financial interest(s): S.Sc. is an employee of AstraZeneca and may own stock or stock options.

\section{ACKNOWLEDGMENTS}

We are grateful for access to an $800 \mathrm{MHz}$ NMR instrument at the Swedish NMR Centre. The Monte Carlo conformational searches for the NAMFIS analyses were performed using resources provided by the Swedish National Infrastructure for Computing (SNIC) at NSC (project 2020-5-435). We thank OpenEye scientific software for a free academic license of OMEGA. We also thank Lianne H. E. Wieske and Stefan Peintner for valuable suggestions on the NAMFIS analysis of 1 and 2. 


\section{ABBREVIATIONS}

IMHB, intramolecular hydrogen bond; NAMFIS, NMR analysis of molecular flexibility in solution; NRotB, number of rotatable bonds; RMSD, root-mean-square deviation; $\mathrm{R}_{\mathrm{gyr}}$, radius of gyration; SMILES, simplified molecular-input lineentry system; SA 3D PSA, solvent accessible 3D polar surface area; TPSA, topological polar surface area

\section{REFERENCES}

(1) Villar, E. A.; Beglov, D.; Chennamadhavuni, S.; Porco, J. A., Jr.; Kozakov, D.; Vajda, S.; Whitty, A. How proteins bind macrocycles. Nat. Chem. Biol. 2014, 10, 723-731.

(2) Doak, B. C.; Zheng, J.; Dobritzsch, D.; Kihlberg, J. How beyond rule of 5 drugs and clinical candidates bind to their targets. J. Med. Chem. 2016, 59, 2312-2327.

(3) Egbert, M.; Whitty, A.; Keseru, G. M.; Vajda, S. Why some targets benefit from beyond rule of five drugs. J. Med. Chem. 2019, 62, $10005-10025$.

(4) Di, L.; Artursson, P.; Avdeef, A.; Benet, L. Z.; Houston, J. B.; Kansy, M.; Kerns, E. H.; Lennernas, H.; Smith, D. A.; Sugano, K. The critical role of passive permeability in designing successful drugs. ChemMedChem 2020, 15, 1862-1874.

(5) Rezai, T.; Bock, J. E.; Zhou, M. V.; Kalyanaraman, C.; Lokey, R. S.; Jacobson, M. P. Conformational flexibility, internal hydrogen bonding, and passive membrane permeability: successful in silico prediction of the relative permeabilities of cyclic peptides. J. Am. Chem. Soc. 2006, 128, 14073-14080.

(6) Price, D. A.; Eng, H.; Farley, K. A.; Goetz, G. H.; Huang, Y.; Jiao, Z.; Kalgutkar, A. S.; Kablaoui, N. M.; Khunte, B.; Liras, S.; Limberakis, C.; Mathiowetz, A. M.; Ruggeri, R. B.; Quan, J. M.; Yang, Z. Comparative pharmacokinetic profile of cyclosporine (CsA) with a decapeptide and a linear analogue. Org. Biomol. Chem. 2017, $15,2501-2506$.

(7) Bogdan, A. R.; Davies, N. L.; James, K. Comparison of diffusion coefficients for matched pairs of macrocyclic and linear molecules over a drug-like molecular weight range. Org. Biomol. Chem. 2011, 9, $7727-7733$

(8) Okumu, F. W.; Pauletti, G. M.; Vander Velde, D. G.; Siahaan, T. J.; Borchardt, R. T. Effect of restricted conformational flexibility on the permeation of model hexapeptides across Caco-2 cell monolayers. Pharm. Res. 1997, 14, 169-175.

(9) Kwon, Y. U.; Kodadek, T. Quantitative comparison of the relative cell permeability of cyclic and linear peptides. Chem. Biol. 2007, 14, 671-677.

(10) Tyagi, M.; Begnini, F.; Poongavanam, V.; Doak, B. C.; Kihlberg, J. Drug syntheses beyond the rule of 5. Chem. - Eur. J. 2020, 26, 4988.

(11) Whitty, A.; Zhong, M.; Viarengo, L.; Beglov, D.; Hall, D. R.; Vajda, S. Quantifying the chameleonic properties of macrocycles and other high-molecular-weight drugs. Drug Discovery Today 2016, 21, $712-717$.

(12) Caron, G.; Ermondi, G. Molecular descriptors for polarity: the need for going beyond polar surface area. Future Med. Chem. 2016, 8, 2013-2016.

(13) Rossi Sebastiano, M.; Doak, B. C.; Backlund, M.; Poongavanam, V.; Over, B.; Ermondi, G.; Caron, G.; Matsson, P.; Kihlberg, J. Impact of dynamically exposed polarity on permeability and solubility of chameleonic drugs beyond the rule of 5. J. Med. Chem. 2018, 61, 4189-4202.

(14) Poongavanam, V.; Atilaw, Y.; Ye, S.; Wieske, L. H. E.; Erdelyi, M.; Ermondi, G.; Caron, G.; Kihlberg, J. Predicting the permeability of macrocycles from conformational sampling - limitations of molecular flexibility. J. Pharm. Sci. 2021, 110, 301-313.

(15) Danelius, E.; Poongavanam, V.; Peintner, S.; Wieske, L. H. E.; Erdelyi, M.; Kihlberg, J. Solution conformations explain the chameleonic behaviour of macrocyclic drugs. Chem. - Eur. J. 2020, $26,5231-5244$
(16) Poongavanam, V.; Danelius, E.; Peintner, S.; Alcaraz, L.; Caron, G.; Cummings, M. D.; Wlodek, S.; Erdelyi, M.; Hawkins, P. C. D.; Ermondi, G.; Kihlberg, J. Conformational sampling of macrocyclic drugs in different environments: can we find the relevant conformations? ACS Omega 2018, 3, 11742-11757.

(17) Witek, J.; Wang, S.; Schroeder, B.; Lingwood, R.; Dounas, A.; Roth, H. J.; Fouche, M.; Blatter, M.; Lemke, O.; Keller, B.; Riniker, S. Rationalization of the membrane permeability differences in a series of analogue cyclic decapeptides. J. Chem. Inf. Model. 2019, 59, 294308.

(18) Le Roux, A.; Blaise, E.; Boudreault, P. L.; Comeau, C.; Doucet, A.; Giarrusso, M.; Collin, M. P.; Neubauer, T.; Kolling, F.; Goller, A. H.; Seep, L.; Tshitenge, D. T.; Wittwer, M.; Kullmann, M.; Hillisch, A.; Mittendorf, J.; Marsault, E. Structure-permeability relationship of semipeptidic macrocycles - understanding and optimizing passive permeability and efflux ratio. J. Med. Chem. 2020, 63, 6774-6783.

(19) Over, B.; Matsson, P.; Tyrchan, C.; Artursson, P.; Doak, B. C.; Foley, M. A.; Hilgendorf, C.; Johnston, S. E.; Lee, M. D. t.; Lewis, R. J.; McCarren, P.; Muncipinto, G.; Norinder, U.; Perry, M. W.; Duvall, J. R.; Kihlberg, J. Structural and conformational determinants of macrocycle cell permeability. Nat. Chem. Biol. 2016, 12, 1065-1074.

(20) Kamenik, A. S.; Kraml, J.; Hofer, F.; Waibl, F.; Quoika, P. K.; Kahler, U.; Schauperl, M.; Liedl, K. R. Macrocycle Cell Permeability Measured by Solvation Free Energies in Polar and Apolar Environments. J. Chem. Inf. Model. 2020, 60, 3508-3517.

(21) Over, B.; McCarren, P.; Artursson, P.; Foley, M.; Giordanetto, F.; Gronberg, G.; Hilgendorf, C.; Lee, M. D. t.; Matsson, P.; Muncipinto, G.; Pellisson, M.; Perry, M. W.; Svensson, R.; Duvall, J. R.; Kihlberg, J. Impact of stereospecific intramolecular hydrogen bonding on cell permeability and physicochemical properties. J. Med. Chem. 2014, 57, 2746-2754.

(22) Begnini, F.; Poongavanam, V.; Over, B.; Castaldo, M.; Geschwindner, S.; Johansson, P.; Tyagi, M.; Tyrchan, C.; Wissler, L.; Sjo, P.; Schiesser, S.; Kihlberg, J. Mining natural products for macrocycles to drug difficult targets. J. Med. Chem. 2021, 64, 10541072.

(23) Artursson, P.; Karlsson, J. Correlation between oral drug absorption in humans and apparent drug permeability coefficients in human intestinal epithelial (Caco-2) cells. Biochem. Biophys. Res. Commun. 1991, 175, 880-885.

(24) Gramse, G.; Dols-Perez, A.; Edwards, M. A.; Fumagalli, L.; Gomila, G. Nanoscale measurement of the dielectric constant of supported lipid bilayers in aqueous solutions with electrostatic force microscopy. Biophys. J. 2013, 104, 1257-1262.

(25) Cicero, D. O.; Barbato, G.; Bazzo, R. Nmr analysis of molecular flexibility in solution - a new method for the study of complex distributions of rapidly exchanging conformations - application to a 13-residue peptide with an 8-residue Loop. J. Am. Chem. Soc. 1995, $117,1027-1033$

(26) Nevins, N.; Cicero, D.; Snyder, J. P. A test of the singleconformation hypothesis in the analysis of NMR data for small polar molecules: A force field comparison. J. Org. Chem. 1999, 64, 39793986.

(27) Thepchatri, P.; Cicero, D. O.; Monteagudo, E.; Ghosh, A. K.; Cornett, B.; Weeks, E. R.; Snyder, J. P. Conformations of laulimalide in DMSO-d $\mathrm{d}_{6}$. J. Am. Chem. Soc. 2005, 127, 12838-12846.

(28) Hawkins, P. C. D., Wlodek, S. OMEGA, version 3.0; OpenEye Scientific Software: Santa Fe, NM, 2016.

(29) Halgren, T. A. Merck molecular force field 0.1. Basis, form, scope, parameterization, and performance of MMFF94. J. Comput. Chem. 1996, 17, 490-519.

(30) Mulligan, V. K. The emerging role of computational design in peptide macrocycle drug discovery. Expert Opin. Drug Discovery 2020, $15,833-852$.

(31) Narang, P.; Bhushan, K.; Bose, S.; Jayaram, B. A computational pathway for bracketing native-like structures fo small alpha helical globular proteins. Phys. Chem. Chem. Phys. 2005, 7, 2364-2375.

(32) Guimarães, C. R.; Mathiowetz, A. M.; Shalaeva, M.; Goetz, G.; Liras, S. Use of 3D properties to characterize beyond rule-of-5 
property space for passive permeation. J. Chem. Inf. Model. 2012, 52, $882-890$.

(33) Ertl, P.; Rohde, B.; Selzer, P. Fast calculation of molecular polar surface area as a sum of fragment-based contributions and its application to the prediction of drug transport properties. J. Med. Chem. 2000, 43, 3714-3717.

(34) Clark, D. E. What has polar surface area ever done for drug discovery? Future Med. Chem. 2011, 3, 469-484.

(35) Palm, K.; Luthman, K.; Ungell, A. L.; Strandlund, G.; Artursson, P. Correlation of drug absorption with molecular surface properties. J. Pharm. Sci. 1996, 85, 32-39.

(36) Caron, G.; Digiesi, V.; Solaro, S.; Ermondi, G. Flexibility in early drug discovery: focus on the beyond-Rule-of- 5 chemical space. Drug Discovery Today 2020, 25, 621-627.

(37) Veber, D. F.; Johnson, S. R.; Cheng, H. Y.; Smith, B. R.; Ward, K. W.; Kopple, K. D. Molecular properties that influence the oral bioavailability of drug candidates. J. Med. Chem. 2002, 45, 26152623.

(38) Kier, L. B. An index of flexibility from molecular shape descriptors. Prog. Clin. Biol. Res. 1989, 291, 105-109. 